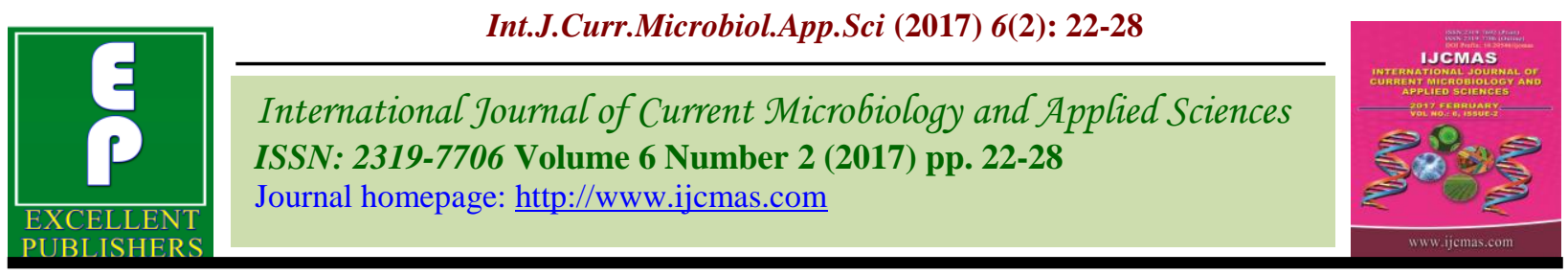

Original Research Article http://dx.doi.org/10.20546/ijcmas2017.602.003

\title{
Potential Donor Parents for Yield and its Associated Components in Kharif Sorghum
}

\author{
Vikas Mangal, R.B. Ghorade*, V.V. Kalpande, Dipali P. Thakare and S.B. Thawari
}

All India Coordinated Sorghum Improvement Project, Akola Centre, Sorghum Research Unit, Dr. Panjabrao Deshmukh Krishi Vidyapeeth, Akola - 444104 (M.S.), India

*Corresponding author

\section{A B S T R A C T}

Keywords

Sorghum, GCA, Combining ability analysis,

Line $\mathrm{x}$ tester.

Article Info

Accepted:

05 January 2017

Available Online:

10 February 2017
The field experiment was carried out at sorghum research Unit, Dr. P.D.K.V., Akola to identify the potential parents in the kharif sorghum. Six lines and eleven testers were crossed in line $\mathrm{x}$ tester fashion and the resultant 66 hybrids were evaluated along with the check (CSH -35) for yield and its associated components for combining ability analysis. The general combining ability study revealed that among the lines, the line AKMS 89 A $\left(4.10^{* *}\right)$ and another line AKMS 103-8-1A (5.19**) were the best general combiners for the grain yield along with some of the desirable yield contributing characters as days to 50 $\%$ flowering $\left(-2.34 * *\right.$ and $-1.40^{* *}$ resp.), days to maturity $(-0.96 * *$ and $-0.57 *$ resp.), number of primaries per panicle $(4.28 * *$ and $6.64 * *$ resp.) and number of grains per panicle (109.03** and $186.81^{* *}$ resp.). AKMS 103-8-1 A had highly significant positive sca effect for fodder yield per plant $\left(5.30^{* *}\right)$. Among the testers, the tester AKR 525(4.68**), AKR 530(3.97**), AKR 524 (3.04**), AKR 528 (2.64*) and AKR 529 $\left(2.33^{*}\right)$ were found to be the best for grain yield per plant. These two lines and five testers need to be extensively used in crossing programme for development of high yielding kharif sorghum hybrids.

\section{Introduction}

Sorghum (Sorghum bicolor L. Moench) is one of the most important staple food and fodder crops in parts of the semi-arid tropics of the world and cultivated in areas considered to be too dry and hot for other cereals, because of its tolerance to drought and heat stress. Sorghum is the $\mathrm{C}_{4}$ plant so, considering the current climate changing scenario it is the important crop of subtropical region with rich diversity and high potential for yield.

It is the third most important grain crop in India, next only to rice and wheat. Maharashtra, Karnataka, Madhya Pradesh,
Andhra Pradesh, Rajasthan, Gujarat and UP are the major sorghum growing states of India.

Hybrid vigour and its commercial exploitation in Kharif sorghum is an important practice in increasing sorghum production (Rana et al., 1997). The estimates of combining ability are useful to predict the relative performance of different lines in hybrid combinations. Selection of parents for hybridization can be made with the help of combining ability analysis (Sprague and Tatum, 1942). Besides its use in selection of parents, it indicates the 
nature and magnitude of various types of gene actions involved in the expression of quantitative characters. Such information is of potential use in formulating and executing an efficient breeding programme for achieving maximum genetic gain with minimum resources and time. Line $x$ tester analysis is a precise method for obtaining such information when a large number of parents to be tested. Keeping this in view, current investigation was carried out under line $\mathrm{x}$ tester analysis to develop suitable hybrid parents in Kharif sorghum. In the development of the high yielding hybrids, the identification of the potential parental lines is of prime importance. General combining analysis is the best tool to assess the potential of any parent. In the present study the attempt was made to recognize such promising parental lines using combining ability analysis.

\section{Materials and Methods}

In current field experiment, six cytoplasmic male sterile (CGMS) lines viz., AKMS 89 A, AKMS 90 A, ICS 279 A, AKMS 103-8 1A, ICS $751 \mathrm{~A}$ and ICS 733 A were crossed with eleven testers viz., AKR 523, AKR 524, AKR 525, AKR 492, AKR 492-1, AKR 526, AKR 527, AKR 528, AKR 529, AKR 530, and AKR 531 were crossed in line $x$ tester fashion during rabi 2014-15 at Sorghum Research Unit, Dr. Panjabrao Deshmukh Krishi Vidyapeeth, Akola.

Further, sixteen parents (six lines + ten testers) and their resulting 66 hybrids along with two standard checks $\mathrm{CSH}-35$ and $\mathrm{CSH}-$ 14 were sown, during kharif 2015-16 in randomized block design with three replications for evaluation. The seed material was treated with tetramethylthiuramdisulphide at $3 \mathrm{~g} / \mathrm{kg}$ prior to sowing to prevent fungal disease infestations while germination. The recommended agronomic and plant protection package of practices adopted to raise the healthy crop growth. The observations were recorded on five randomly selected plants per plot per replication for plant height $(\mathrm{cm})$, panicle weight $(\mathrm{g})$, panicle length $(\mathrm{cm})$, panicle breadth $(\mathrm{cm})$, number of primaries per panicle, number of secondaries per panicle, number of grains per panicle, 1000 seed weight (g), grain hardness $\left(\mathrm{kg} / \mathrm{cm}^{2}\right)$, grain yield per plant $(\mathrm{g})$ and fodder yield per plant $(\mathrm{g})$.

For days to $50 \%$ flowering and days to maturity observations were recorded on plot basis. Shoot fly dead heart observation was recorded on percentage basis. The collected data for all above characters subjected to combining ability analysis and to identify the potential donor parents for grain yield in kharif grain sorghum by following Kempthorne (1957) method.

\section{Results and Discussion}

Analysis of variance for combining ability is presented in the table 1 . The total variance due to hybrids was partitioned into portions attributable to lines (females), testers (males), their interaction (lines $\mathrm{x}$ testers) and error sources. The variation due to lines $\mathrm{x}$ testers showed highly significant differences for all the yield and yield associated characters. The components of variances attributable to lines and testers were used as a measure of general combining ability.

The estimates of general combining ability effects of the lines and testers are presented in table 2 and table 3 respectively. Among the six lines, the line AKMS 103-8-1A has been identified as the best general combiner for yield per plant $(5.19 * *)$ along with nine other component characters i.e. days to 50 per cent flowering $\left(-1.40^{* *}\right)$, days to maturity ($\left.0.57^{*}\right)$, plant height $\left(3.64^{* *}\right)$, panicle weight $\left(5.91^{* *}\right)$, panicle length $\left(2.49^{* *}\right)$, number of primaries per panicle $(6.64 * *)$, number of secondaries per panicle $(38.48 * *)$, number of grains per panicle $\left(186.81^{* *}\right)$ and fodder yield per plant $\left(5.30^{* *}\right)$. 
Table.1 Analysis of variance (ANOVA) for combining ability for various yield and yield contributing characters in sorghum

\begin{tabular}{|c|c|c|c|c|c|c|c|c|c|c|c|c|c|c|c|}
\hline $\begin{array}{l}\text { Source of } \\
\text { Variation }\end{array}$ & d.f. & $\begin{array}{c}\text { Days to } \\
\mathbf{5 0 \%} \\
\text { Flowering }\end{array}$ & $\begin{array}{l}\text { Day to } \\
\text { maturity }\end{array}$ & $\begin{array}{l}\text { Plant } \\
\text { Height } \\
\text { (cm) }\end{array}$ & $\begin{array}{c}\text { Panicle } \\
\text { Weight } \\
\text { (gm) }\end{array}$ & $\begin{array}{c}\text { Panicle } \\
\text { Length } \\
\text { (cm) }\end{array}$ & $\begin{array}{c}\text { Panicle } \\
\text { breadth } \\
\text { (cm) }\end{array}$ & $\begin{array}{c}\text { Number of } \\
\text { primaries } \\
\text { per } \\
\text { panicle }\end{array}$ & $\begin{array}{l}\text { Number of } \\
\text { secondaries } \\
\text { per panicle }\end{array}$ & $\begin{array}{c}\text { Number of } \\
\text { Grains/ } \\
\text { Panicle }\end{array}$ & $\begin{array}{c}\text { Grain } \\
\text { Yield/ } \\
\text { Plant (g) }\end{array}$ & $\begin{array}{c}1000 \\
\text { Seed } \\
\text { Weight } \\
\text { (g) }\end{array}$ & $\begin{array}{c}\text { Grain } \\
\text { Hardness } \\
\left(\mathbf{k g} / \mathbf{c m}^{2}\right)\end{array}$ & $\begin{array}{l}\text { Fodder } \\
\text { Yield/ } \\
\text { Plant (g) }\end{array}$ & $\begin{array}{l}\text { Shoot fly } \\
\text { Dead heart } \\
\text { percentage } \\
\text { (at } 28 \text { days) }\end{array}$ \\
\hline & & 1 & 2 & 3 & 4 & 5 & 6 & 7 & 8 & 9 & 10 & 11 & 12 & 13 & 14 \\
\hline Replications & 2 & 2.68 & $7.15^{*}$ & 12.97 & $9.01 *$ & 3.17 & 0.72 & 1.35 & $436.7 *$ & 17866.36 & 15.38 & 0.74 & $1.84^{*}$ & 27.05 & 1.89 \\
\hline Lines & 5 & $83.48 * *$ & 20.02 & 469.49 & 1355.44 & $82.42 *$ & $3.33^{*}$ & $747.63^{* *}$ & $25180.57 * *$ & $554527.26^{* *}$ & $585.11^{*}$ & $50.61 *$ & 7.22 & 1062.19 & 4.42 \\
\hline Testers & 10 & 27.96 & 23.72 & 526.56 & 575.86 & 25.77 & 0.63 & 276.16 & 9296.27 & 205002.51 & 245.71 & 31.8 & 0.9 & $4103.56^{*}$ & 6.72 \\
\hline Line $\mathrm{x}$ Testers & 50 & $14.23 * *$ & $13.57 * *$ & $525.75 * *$ & $949.6^{* *}$ & $29.32 * *$ & $1.21 * *$ & $181.85^{* *}$ & $6116.37 * *$ & $130543.01 * *$ & $211.43 * *$ & $17.39^{* *}$ & $3.22 * *$ & $2010.27 * *$ & $4.73 * *$ \\
\hline Error & 130 & 3.45 & 1.72 & 40.6 & 2.92 & 1.44 & 0.26 & 2.69 & 117.44 & 6245.06 & 28.82 & 0.82 & 0.42 & 43.24 & 1.80 \\
\hline
\end{tabular}

Note: $*$ - significant at $5 \%$ level of significance, $* *$ - significant at $1 \%$ level of significance

Table.2 Estimates of general combining ability (GCA) effects of lines for various characters under line $\mathrm{x}$ tester analysis

\begin{tabular}{|c|c|c|c|c|c|c|c|c|c|c|c|c|c|c|c|}
\hline $\begin{array}{l}\text { Sr. } \\
\text { No. }\end{array}$ & Parents & $\begin{array}{c}\text { Days to } \\
\mathbf{5 0 \%} \\
\text { Flowering }\end{array}$ & $\begin{array}{c}\text { Days } \\
\text { to } \\
\text { Maturity }\end{array}$ & $\begin{array}{l}\text { Plant } \\
\text { Height } \\
\text { (cm) }\end{array}$ & $\begin{array}{l}\text { Panicle } \\
\text { Weight } \\
\text { (g) }\end{array}$ & $\begin{array}{l}\text { Panicle } \\
\text { Length } \\
\text { (cm) }\end{array}$ & $\begin{array}{c}\text { Panicle } \\
\text { Breadth } \\
\text { (cm) }\end{array}$ & $\begin{array}{c}\begin{array}{c}\text { Number } \\
\text { of } \\
\text { Primaries/ } \\
\text { Panicle }\end{array} \\
\end{array}$ & $\begin{array}{c}\text { Number of } \\
\text { Secondaries/Plant }\end{array}$ & $\begin{array}{c}\text { Number } \\
\text { of } \\
\text { Grains/ } \\
\text { Panicle }\end{array}$ & $\begin{array}{c}\text { Grain } \\
\text { Yield/ } \\
\text { Plant } \\
\text { (g) }\end{array}$ & $\begin{array}{c}1000 \\
\text { Seed } \\
\text { Weight } \\
\text { (g) }\end{array}$ & $\begin{array}{c}\text { Grain } \\
\text { Hardness }\end{array}$ & $\begin{array}{c}\text { Fodder } \\
\text { Yield } \\
\text { /Plant } \\
\text { (g) } \\
\end{array}$ & $\begin{array}{c}\text { Shoot Fly } \\
\text { Dead } \\
\text { Heart } \\
(\%)\end{array}$ \\
\hline & & 1 & 2 & 3 & 4 & 5 & 6 & 7 & 8 & 9 & 10 & 11 & 12 & 13 & 14 \\
\hline \multicolumn{16}{|c|}{ Lines (Females) } \\
\hline 1 & AKMS 89 A & $-2.34 * *$ & $-0.96^{* *}$ & $-5.02 * *$ & $-2.41 * *$ & $-1.30 * *$ & -0.12 & $4.28^{* *}$ & $24.85^{* * *}$ & $109.03 * *$ & $4.10^{* *}$ & -0.09 & 0.10 & -2.10 & -0.27 \\
\hline 2 & AKMS $90 \mathrm{~A}$ & 0.51 & $0.71^{* *}$ & -0.69 & $-10.03 * *$ & $-2.04 * *$ & $-0.39 * *$ & $-6.06^{* *}$ & $-35.23 * *$ & $-166.02 * *$ & $-5.55^{* * *}$ & 0.20 & $-0.47 * *$ & $-8.10 * *$ & 0.37 \\
\hline 3 & ICS 279 A & 0.21 & $-0.54 *$ & 1.91 & $-3.33 * *$ & -0.02 & $-0.23 * *$ & $-3.25 * *$ & $-18.88 * *$ & $-85.17 * *$ & $-3.38 * *$ & $-0.50 * *$ & $0.65 * *$ & $4.75^{* *}$ & 0.46 \\
\hline 4 & AKMS103-8 1A & $-1.40 * *$ & $-0.57^{*}$ & $3.64 * *$ & $5.91 * *$ & $2.49 * *$ & 0.16 & $6.64 * *$ & $38.48^{* *}$ & $186.81 * *$ & $5.19^{* *}$ & $-0.59 * *$ & $-0.6^{* *}$ & $5.30 * *$ & -0.23 \\
\hline 5 & ICS 751 A & $1.09 * *$ & $0.89 * *$ & $-3.69 * *$ & $3.46 * *$ & 0.31 & 0.08 & $-1.86 * *$ & $-10.80 * *$ & $-48.99 * *$ & -1.32 & $-1.337 * *$ & $0.29 *$ & $-4.40 * *$ & -0.43 \\
\hline \multirow[t]{4}{*}{6} & ICS 733 A & $1.93^{* *}$ & $0.465^{*}$ & $3.85 * *$ & $6.40 * *$ & $0.57 * *$ & $0.50 * *$ & 0.27 & 1.58 & 4.34 & 0.96 & $2.296 * *$ & 0.03 & $4.57 * *$ & 0.10 \\
\hline & $\mathrm{SE}(\mathrm{gi}) \pm$ & 0.90 & 0.61 & 1.18 & 0.30 & 0.20 & 0.09 & 0.29 & 1.91 & 14.01 & 0.87 & 0.16 & 0.11 & 1.16 & 0.62 \\
\hline & $\mathrm{CD}$ at $5 \%$ & 0.34 & 0.23 & 2.33 & 0.59 & 0.40 & 0.17 & 0.57 & 3.78 & 27.72 & 1.71 & 0.32 & 0.23 & 2.29 & 0.24 \\
\hline & $\mathrm{CD}$ at $1 \%$ & 0.68 & 0.46 & 3.09 & 0.78 & 0.53 & 0.23 & 0.76 & 4.99 & 36.63 & 2.26 & 0.42 & 0.30 & 3.02 & 0.47 \\
\hline
\end{tabular}

Note: * - significant at $5 \%$ level of significance, $* *$ - significant at $1 \%$ level of significance 


\section{Int.J.Curr.Microbiol.App.Sci (2017) 6(2): 22-28}

Table.3 Estimates of general combining ability (GCA) effects of testers for various yield characters under line $\mathrm{x}$ tester analysis

\begin{tabular}{|c|c|c|c|c|c|c|c|c|c|c|c|c|c|c|c|}
\hline $\begin{array}{l}\text { Sr. } \\
\text { No. }\end{array}$ & Parents & $\begin{array}{c}\text { Days to } \\
50 \% \\
\text { Flowering }\end{array}$ & $\begin{array}{c}\text { Days } \\
\text { to } \\
\text { Maturity }\end{array}$ & $\begin{array}{c}\text { Plant } \\
\text { Height } \\
(\mathrm{cm})\end{array}$ & $\begin{array}{c}\text { Panicle } \\
\text { Weight } \\
\text { (g) }\end{array}$ & $\begin{array}{c}\text { Panicle } \\
\text { Length } \\
(\mathrm{cm})\end{array}$ & $\begin{array}{c}\text { Panicle } \\
\text { Breadth } \\
(\mathrm{cm})\end{array}$ & $\begin{array}{c}\begin{array}{c}\text { Number } \\
\text { of }\end{array} \\
\text { Primaries/ } \\
\text { Panicle }\end{array}$ & $\begin{array}{c}\text { Number of } \\
\text { Secondaries/Panicle }\end{array}$ & $\begin{array}{c}\text { Number } \\
\text { of } \\
\text { Grains/ } \\
\text { Panicle }\end{array}$ & $\begin{array}{c}\text { Grain } \\
\text { Yield/ } \\
\text { Plant } \\
(\mathrm{g})\end{array}$ & $\begin{array}{c}1000 \\
\text { Seed } \\
\text { Weight } \\
(\mathrm{g})\end{array}$ & $\begin{array}{c}\text { Grain } \\
\text { Hardness }\end{array}$ & $\begin{array}{c}\text { Fodder } \\
\text { Yield } \\
\text { /Plant } \\
\text { (g) }\end{array}$ & $\begin{array}{c}\text { Shoot Fly } \\
\text { Dead } \\
\text { Heart } \\
(\%)\end{array}$ \\
\hline & & 1 & 2 & 3 & 4 & 5 & 6 & 7 & 8 & 9 & 10 & 11 & 12 & 13 & 14 \\
\hline \multicolumn{16}{|c|}{ Testers (Males) } \\
\hline 1 & AKR 523 & 0.09 & $1.66 * *$ & -0.67 & $-6.77 * *$ & -0.49 & 0.17 & $-5.07 * *$ & $-29.41 * *$ & -139.19 ** & $-4.74 * *$ & 0.57 ** & 0.01 & $-7.21 * *$ & 0.60 \\
\hline 2 & AKR 524 & -0.85 & $-0.90 * *$ & $-8.72 * *$ & -0.23 & $-1.47 * *$ & $-0.38 * *$ & $2.71 * *$ & $15.75 * *$ & $67.26 * *$ & $3.04 *$ & -0.12 & $-0.31 *$ & $-27.11 * *$ & -0.14 \\
\hline 3 & AKR 525 & $2.76 * *$ & $1.49 * *$ & $3.28 *$ & $4.33 * *$ & -0.03 & 0.05 & $6.13 * *$ & $35.58 * *$ & $178.12 * *$ & $4.68 * *$ & $-0.56 *$ & -0.09 & $8.61 * *$ & 0.52 \\
\hline 4 & AKR 492 & -0.52 & $-2.07 * *$ & 1.56 & $-8.14 * *$ & $0.68 *$ & 0.15 & $-6.78 * *$ & $-39.33 * *$ & $-173.44 * *$ & $-6.12 * *$ & $-1.20 * *$ & $0.47 * *$ & $-9.06 * *$ & $0.97 * *$ \\
\hline 5 & AKR 492-1 & -0.74 & $-0.96 * *$ & 0.44 & $0.93 *$ & -0.30 & -0.07 & $-1.46 * *$ & $-8.50 * *$ & $-43.98 *$ & -0.86 & -0.05 & -0.13 & $3.40 *$ & -0.19 \\
\hline 6 & AKR 526 & $-1.52 * *$ & -0.51 & $3.28 *$ & $1.81 * *$ & -0.25 & -0.13 & $-4.17 * *$ & $-24.20 * *$ & $-116.28 * *$ & $-3.85 * *$ & $0.74 * *$ & -0.01 & $15.08 * *$ & 0.02 \\
\hline 7 & AKR 527 & -0.08 & $1.10 * *$ & -1.83 & $6.65 * *$ & -0.12 & -0.09 & $2.07 * *$ & $12.01 * *$ & $50.49 * *$ & -1.10 & 0.05 & 0.24 & $20.21 * *$ & 0.02 \\
\hline 8 & AKR 528 & $-1.19 *$ & 0.04 & $-6.94 * *$ & $2.25 * *$ & $1.58 * *$ & -0.20 & $1.56 * *$ & $9.13 * *$ & 35.94 & $2.64 *$ & $3.21 * *$ & -0.08 & $-18.67 * *$ & $-0.87 * *$ \\
\hline 9 & AKR 529 & 0.15 & $0.66 *$ & 2.06 & $3.21 * *$ & $1.54 * *$ & 0.17 & 0.72 & 4.18 & 20.59 & $2.33 *$ & $-1.79 * *$ & 0.19 & -0.01 & $-0.77 *$ \\
\hline 10 & AKR 530 & $1.59 * *$ & -0.57 & $11.11 * *$ & $5.63 * *$ & $1.13^{* *}$ & 0.23 & $2.80 * *$ & $16.21 * *$ & $84.80 * *$ & $3.97 * *$ & $-1.18 * *$ & -0.04 & 18.14 ** & 0.54 \\
\hline \multirow[t]{4}{*}{11} & AKR 531 & 0.31 & 0.04 & $-3.56 *$ & $-9.67 * *$ & $-2.27 * *$ & 0.08 & $1.48 * *$ & $8.57 * *$ & 35.69 & 0.09 & 0.320 & -0.24 & $-3.34 *$ & $-0.68^{*}$ \\
\hline & $\mathrm{SE}(\mathrm{gi}) \pm$ & 1.22 & 0.82 & 1.60 & 0.40 & 0.27 & 0.12 & 0.39 & 2.58 & 18.97 & 1.17 & 0.22 & 0.16 & 1.57 & 0.85 \\
\hline & $\mathrm{CD}$ at $5 \%$ & 0.47 & 0.31 & 3.16 & 0.80 & 0.54 & 0.23 & 0.78 & 5.11 & 37.54 & 2.32 & 0.43 & 0.31 & 3.10 & 0.32 \\
\hline & $\mathrm{CD}$ at $1 \%$ & 0.92 & 0.62 & 4.18 & 1.06 & 0.71 & 0.31 & 1.03 & 6.75 & 49.60 & 3.06 & 0.57 & 0.41 & 4.09 & 0.64 \\
\hline
\end{tabular}

Note: * significant at $5 \%$ level of significance, $\quad * *$ - significant at $1 \%$ level of significance 


\section{Int.J.Curr.Microbiol.App.Sci (2017) 6(2): 22-28}

Table.4 GCA effects of promising parents in desirable direction for grain yield and other yield contributing characters in sorghum.

\begin{tabular}{|c|c|c|c|c|c|c|c|c|c|c|c|c|c|c|}
\hline Parents & $\begin{array}{c}\text { Days to } 50 \% \\
\text { Flowering }\end{array}$ & $\begin{array}{c}\text { Days to } \\
\text { Maturity }\end{array}$ & $\begin{array}{l}\text { Plant } \\
\text { Height } \\
(\mathrm{cm})\end{array}$ & $\begin{array}{c}\text { Panicle } \\
\text { weight } \\
\text { (g) }\end{array}$ & $\begin{array}{c}\text { Panicle } \\
\text { Length } \\
\text { (cm) }\end{array}$ & $\begin{array}{c}\text { Panicle } \\
\text { Breadth } \\
\text { (cm) }\end{array}$ & $\begin{array}{c}\begin{array}{c}\text { Number } \\
\text { of }\end{array} \\
\text { Primaries/ } \\
\text { Panicle }\end{array}$ & $\begin{array}{c}\text { Number of } \\
\text { secondaries } \\
\text { / panicle }\end{array}$ & $\begin{array}{c}\text { Number } \\
\text { of } \\
\text { Grains/ } \\
\text { Panicle }\end{array}$ & $\begin{array}{c}\text { Grain } \\
\text { Yield/ } \\
\text { Plant (g) }\end{array}$ & $\begin{array}{l}1000 \text { Seed } \\
\text { Weight (g) }\end{array}$ & $\begin{array}{c}\text { Grain } \\
\text { hardness }\end{array}$ & $\begin{array}{c}\text { Fodder } \\
\text { yield per } \\
\text { Plant (g) }\end{array}$ & $\begin{array}{c}\text { Shoot fly dead } \\
\text { heart } \\
\text { percentage (at } \\
28 \text { days) }\end{array}$ \\
\hline & 1 & 2 & 3 & 4 & 5 & 6 & 7 & 8 & 9 & 10 & 11 & 12 & 13 & 14 \\
\hline \multicolumn{15}{|c|}{ Lines (Females) } \\
\hline AKMS 89 A & $-2.34 * *$ & $-0.96 * *$ & $-5.02 * *$ & $-2.41 * *$ & $-1.30 * *$ & -0.12 & $4.28 * *$ & $24.85^{* *}$ & $109.03^{* *}$ & $4.10^{* *}$ & -0.09 & 0.10 & -2.10 & -0.27 \\
\hline AKMS 103-8-1A & $-1.40 * *$ & $-0.57 *$ & $3.64 * *$ & $5.91 * *$ & $2.49^{* *}$ & 0.16 & $6.64 * *$ & $38.48 * *$ & $186.81^{* *}$ & $5.19^{* *}$ & $-0.59 * *$ & $-0.6^{* *}$ & $5.30 * *$ & -0.23 \\
\hline \multicolumn{15}{|c|}{ Testers (Males) } \\
\hline AKR 524 & -0.85 & $-0.90 * *$ & $-8.72 * *$ & -0.23 & $-1.47 * *$ & $-0.38 * *$ & $2.71 * *$ & $15.75^{* *}$ & $67.26^{* *}$ & $3.04 *$ & -0.12 & $-0.31 *$ & $-27.11 * *$ & -0.14 \\
\hline AKR 525 & $2.76^{* * *}$ & $1.49^{* *}$ & $3.28 *$ & $4.33^{* *}$ & -0.03 & 0.05 & $6.13 * *$ & $35.58 * *$ & $178.12 * *$ & $4.68^{* *}$ & $-0.56 *$ & -0.09 & $8.61 * *$ & 0.52 \\
\hline AKR 528 & $-1.19^{*}$ & 0.04 & $-6.94 * *$ & $2.25^{* *}$ & $1.58 * *$ & -0.20 & $1.56^{* *}$ & $9.13^{* *}$ & 35.94 & $2.64 *$ & $3.21 * *$ & -0.08 & $-18.67^{* *}$ & $-0.87 * *$ \\
\hline AKR 529 & 0.15 & $0.66^{*}$ & 2.06 & $3.21 * *$ & $1.54 * *$ & 0.17 & 0.72 & 4.18 & 20.59 & $2.33 *$ & $-1.79 * *$ & 0.19 & -0.01 & $-0.77 *$ \\
\hline AKR 530 & $1.59^{* *}$ & -0.57 & $11.1^{* *}$ & $5.63^{* *}$ & $1.13 * *$ & 0.23 & $2.80 * *$ & $16.21^{* *}$ & $84.80 * *$ & $3.97 * *$ & $-1.18 * *$ & -0.04 & $18.14^{* *}$ & 0.54 \\
\hline
\end{tabular}

Note: $*$ - significant at $5 \%$ level of significance $* *$ - significant at $1 \%$ level of significance 
Fig.1 GCA effects of promising parents in desirable direction for grain yield

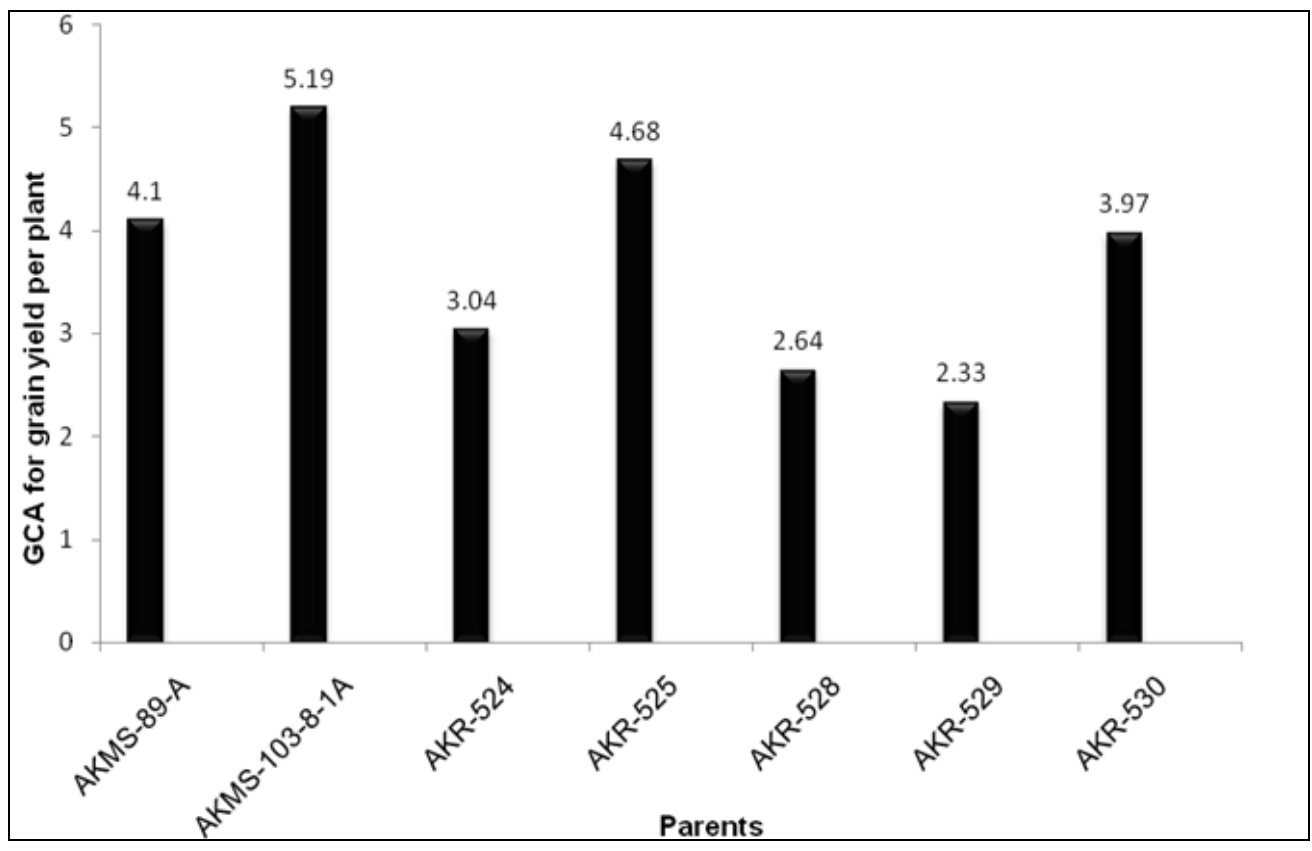

Further, another line AKMS 89 A had given highly significant and desirable GCA effects for grain yield per plant $\left(4.10^{* *}\right)$ along with other five component character i.e. days to 50 per cent flowering $(-2.34 * *)$, days to maturity $\left(-0.96^{* *}\right)$, number of primaries per panicle $\left(4.28^{* *}\right)$, number of secondaries per panicle $\left(24.85^{* *}\right)$ and number of grains per panicle $(109.03 * *)$. Besides these two lines, AKMS 279 A and ICS 751 had significantly high GCA effect for the grain hardness $\left(0.65^{* *}\right.$ and $0.29 *$ respectively.) ultimately which help in the imparting the gain mold resistance in the succeeding genotypes.

Among the ten testers, AKR 525 exhibited highly significant and desirable GCA effects for grain yield per plant (4.68*) along with other desirable characters likes plant height $\left(3.28^{*}\right)$, panicle weight $\left(4.33^{* *}\right)$, number of primaries per panicle $\left(6.13^{* *}\right)$, number of secondaries per panicle $(35.58 * *)$, number of grains per panicle $(178.12 * *)$ and fodder yield per plant $\left(8.61^{* *}\right)$. Similarly, tester AKR 530 showed highly significant positive GCA effects for grain yield per plant $\left(3.97^{* *}\right)$ coupled with yield component characters as plant height $\left(11.11^{* *}\right)$, panicle weight $\left(5.63^{* *}\right)$, panicle length $\left(1.13^{* *}\right)$, number of primaries per panicle $\left(2.80^{* *}\right)$, number of secondaries per panicle $\left(16.21^{* *}\right)$, number of grains per panicle $\left(84.80^{* *}\right)$ and fodder yield per plant $\left(18.14^{* *}\right)$.

With respect to tester AKR 524 significant positive GCA effect for grain yield per plant obtained (3.04*) along with essential yield contributing characters like days to maturity $\left(-0.90^{* *}\right)$, number of primaries per panicle $\left(2.71^{* *}\right)$, number of secondaries per panicle $\left(15.75^{* *}\right)$ and number of grains per panicle $\left(67.26^{* *}\right)$. On the basis of significant positive GCA effects, AKR 528 obtained fourth position which showed for grain yield per plant $(2.64 *)$ along with component characters days to 50 per cent flowering ($\left.1.19^{*}\right)$, panicle weight $\left(2.25^{* *}\right)$, panicle length $\left(1.58^{* *}\right)$, number of primaries per panicle $\left(1.56^{* *}\right)$, number of secondaries per panicle $\left(9.13^{* *}\right), 1000$ seed weight $\left(3.21^{* *}\right)$ 
and shoot fly dead heart percentage $(-0.87 * *)$ with respect to other ten testers. Similarly, tester AKR 529 recorded significant positive GCA effect for grain yield per plant $\left(2.33^{*}\right)$ along with other desirable characters as panicle weight $\left(3.21^{* *}\right)$, panicle length $(1.54 * *)$ and shoot fly dead heart percentage $(-0.77 * *)$ as compare to other testers.

Prabhakar and Raut, (2010); Ghorade et al., (2014) and Kalpande et al., (2015) also reported the promising general combiners for yield and its other important components from their studies. Dar et al., (2015) reported promising parents in maize based on general combining ability effects.

The results of present study indicated that, among six lines, AKMS 89 A (4.10**), AKMS 103-8-1A (5.19**) and ten testers, AKR 525(4.68**), AKR 530(3.97**), AKR 524 (3.04**), AKR 528 (2.64*) and AKR 529 $\left(2.33^{*}\right)$ showed superior performance on the basis of GCA effect (Fig. 1). Further, AKR 528, AKR 529 and AKR 531 showed significant negative GCA effects $(-0.87 * *$, $0.77 *$ and $-0.68^{*}$ respectively) for shoot fly dead hearts which indicated that these testers had shoot fly tolerance ability.

Tester AKR 492 exhibited significant positive GCA effect $(0.47 * *)$ for grain hardness which could be help in the grain mold resistance of genotypes. Therefore, it is need to extensively use these diverse lines as well as diverse testers as donor parental lines in hybridization programme of sorghum to develop high yielding hybrids in kharif sorghum.

\section{References}

Dar, Z.A., Lone, A.A., Alaie, B., Gowhar Ali, Asima Gazal and Abidi, I. 2015. Estimation of combining ability involving quality protein maize (QPM) inbreds under temperate conditions. The Bioscan., 10(2): 863-867.

Ghorade, R.B., Kalpande, V.V., Bhongle, S.A., and Band, P.A. 2014. Combining analysis for drought tolerance and grain yield in rabi sorghum. Int. J. Agri. Sci., 10(1): 344-347.

Kalpande, V.V., Sakhare, B.A., Ghorade, R.B. and Lad, A.P. 2015. Potential parents for yield and its components in rabi sorghum. The Bioscan., 10(2): 837839.

Kempthorne, O. 1957. An Introduction to genetic statistics, John Willey and Sons. Increased. New York, 468-470.

Prabhakar and Raut, M.S. 2010. Exploitation of heterosis using diverse parental lines in rabi sorghum. Electronic J. Plant Breeding, 1(4): 680-684.

Rana, B.S., Kaul, S. and Rao, M.H. 1997. Impact of genetic improvement on sorghum productivity in India. In "Proceedings of the International Conference on Genetic Improvement of Sorghum and Pearl millet', 22-27 September 1996, Holiday Inn Plaza, Lubbock, Texas. Pp. 142165.

Sprague, G.F. and Tatum L.A. 1942. General $v s$. specific combining ability in single crosses of corn. J. Amer. Soc. Agron., 34: 923-932.

\section{How to cite this article:}

Vikas Mangal, R.B. Ghorade, V.V. Kalpande, Dipali P. Thakare and Thawari, S.B. 2017. Potential Donor Parents for Yield and its Associated Components in Kharif Sorghum. Int.J.Curr.Microbiol.App.Sci. 6(2): 22-28. doi: http://dx.doi.org/10.20546/ijcmas.2017.602.003 